\title{
Überlegungen zur Liberalisierung des europäischen Verteidigungsguitermarktes
}

\author{
Xavier Pacreau und Andreas Schwab*
}

Der europäische Verteidigungsgütermarkt ist heute immer noch stark in Einzelmärkte unterteilt. Dabei wird häufig gegen die Regeln der Gemeinschaft über die öffentlichen Beschaffungsmärkte verstoßen, indem Art. 296 des EG-Vertrags (EGV) äußerst weit ausgelegt wird. ${ }^{1}$ Diese Vorgehensweise hat zu einer quasi ständigen Anwendung der verschiedenen nationalen Regeln geführt, die darüber hinaus auch noch relativ unverständlich sind. Dadurch kommt es zu einer Abschottung der öffentlichen Märkte im Bereich der Verteidigungsgüter, was einen wirklich europäischen Wettbewerb verhindert. In dieser Situation stellt sich die Frage nach der Öffnung des europäischen Rüstungsmarkts zur Schaffung wirklichen Wettbewerbs und weitgehender Transparenz.

Es sind im Wesentlichen drei Gesichtspunkte, die die Liberalisierung des Marktes für Verteidigungsgüter unerlässlich machen. Erstens die Verkleinerung der Verteidigungshaushalte $^{2}$ bei gleichzeitigem Anstieg der Kosten für Rüstungsprogramme. Dies führt dazu, dass Bestellungen verschoben werden und die Anzahl der Programme zurückgefahren wird. Hinzu kommt, dass es für Firmen unmöglich ist, ausreichende Synergieeffekte zur Senkung der Produktionskosten allein auf der Basis der nationalen Nachfrage durchzuführen. Zweitens ist die Liberalisierung eine logische Folge der Entscheidung der EU-Mitgliedstaaten, eine Europäische Sicherheits- und Verteidigungspolitik (ESVP) zu entwickeln. Diese setzt die Aufrechterhaltung einer gemeinsamen industriellen und technologischen Grundlage (EDITB, aus dem Englischen: European Defence Industrial and Technological Base) für den Bereich der Verteidigung und die Gewährleistung der Versorgungssicherheit voraus. Drittens die Notwendigkeit einer Konsolidierung im Bereich der Rüstungsunternehmen auf europäischer Ebene, um angesichts der internationalen Konkurrenz wettbewerbsfähig zu bleiben. Diese drei Faktoren erfordern gleichzeitig die Bildung von transeuropäischen Firmengruppen auf den Gebieten, wo sie noch nicht existieren, eine Vereinheitlichung des europäischen Marktes für Verteidigungsgüter durch eine genauere Eingrenzung der Ausnahmeregelungen des Art. 296 EGV und eventuell sogar die Harmonisierung und Angleichung der Vergaberegelungen in den Verteidigungsmärkten innerhalb der Europäischen Union.

* Xavier Pacreau, D.E.A, Geschäftsführer der deutsch-französischen Zeitschrift „Le Forum“, Paris.

Dr. Andreas Schwab, MdEP, Vorsitzender des deutsch-französischen Forum e.V., Freiburg, binnenmarktpolitischer Sprecher der CDU/CSU-Gruppe im Europäischen Parlament, Brüssel.

1 Art. 296 EGV (ex-Art. 223)

(1) Die Vorschriften dieses Vertrags stehen folgenden Bestimmungen nicht entgegen:

a) Ein Mitgliedstaat ist nicht verpflichtet, Auskünfte zu erteilen, deren Preisgabe seines Erachtens seinen wesentlichen Sicherheitsinteressen widerspricht;

b) jeder Mitgliedstaat kann die Maßnahmen ergreifen, die seines Erachtens für die Wahrung seiner wesentlichen Sicherheitsinteressen erforderlich sind, soweit sie die Erzeugung von Waffen, Munition und Kriegsmaterial oder den Handel damit betreffen; diese Maßnahmen dürfen auf dem Gemeinsamen Markt die Wettbewerbsbedingungen hinsichtlich der nicht eigens für militärische Zwecke bestimmten Waren nicht beeinträchtigen.

2 Die Verringerung der Verteidigungshaushalte betrug zwischen 1993 und 1998: 16,4 Prozent in Deutschland, 14,1 Prozent in Frankreich, 13,8 Prozent in Großbritannien und 35,1 Prozent in Italien. Vgl. dazu Französische Kommission für Verteidigung und Streitkräfte: Bericht Nr. 42, 27.09.2005. 
Es bleibt nun die Aufgabe der Kommission genauer zu formulieren, welcher Teil des Markts für Verteidigungsgüter nicht den gemeinsamen europäischen Regeln unterworfen werden soll. Diese Klarstellung muss präzise genug sein, um die Inanspruchnahme der Ausnahmeregelung des Art. 296 EGV auf wirkliche Sonderfälle zu begrenzen. Die Analyse der Beiträge und Wechselwirkungen zwischen den Regelungsansätzen gemeinschaftsrechtlicher (Richtlinien) und intergouvernementaler Natur (Verhaltenskodizes) wird es letztendlich erlauben, den Rahmen und die Modalitäten des Öffnungsprozesses genauer zu beurteilen.

\section{Die Öffnung des Verteidigungsgütermarktes und die Auslegung von Art. 296 EGV}

Die Öffnung des europäischen Verteidigungsgütermarktes stellt gewisse Anforderungen an die nationalen Verteidigungsmärkte. Zuerst muss der Marktzugang für alle EU-Unternehmen gewährleistet werden, damit sie an den Ausschreibungen aller Mitgliedstaaten teilnehmen können. Hierfür sind Regelungen zur Sicherung von Wettbewerb und Transparenz zu treffen.

Es sind im Wesentlichen drei Initiativen, die die Öffnung der Märkte zum Ziel haben. Die Eine ist zwischenstaatlicher Natur: Der Verhaltenskodex über die Beschaffung von Verteidigungsgütern ${ }^{3}$. Die beiden Anderen sind gemeinschaftsrechtlicher Natur: Die Mitteilung der Kommission bezüglich der Anwendung des Artikels 296 EGV auf die Beschaffung von Verteidigungsgütern ${ }^{4}$ und die Richtlinie für die Beschaffung von militärischen Ausrüstungen 5 .

Jedoch versuchen nur die beiden gemeinschaftlichen Instrumente die Praxis der Mitgliedstaaten wirklich grundlegend zu verändern, indem die Tragweite der Ausnahmeregelung von Art. 296 EGV geklärt wird und die Vergabe vereinheitlicht werden soll. Es sind also die Initiativen der Kommission, die entscheiden werden, auf welche Art und Weise die Märkte geöffnet werden, um einen europäischen Markt für Verteidigungsgüter zu schaffen.

\section{Vergemeinschaftung eines bedeutenden Anteils des europäischen Verteidigungsgüter- marktes}

Aufgrund des Gemeinschaftsrechts sind die Regeln des Binnenmarktes grundsätzlich auch auf Verträge anzuwenden, welche Produkte und Dienstleistungen in der Verteidigungsgüterbranche betreffen. ${ }^{6}$ Dennoch behält es Art. 296 EGV den Mitgliedstaaten vor, die gemeinsamen Regeln für den öffentlichen Markt zu umgehen, falls ,,wesentliche Sicherheitsinteressen“ auf den Gebieten, ,die sich auf die Herstellung oder den Handel mit Waffen, Munition oder Kriegsmaterial beziehen“, gefährdet werden könnten.

In der Praxis werden die Verteidigungsmärkte unter Berufung auf die Ausnahmemöglichkeiten des Art. 296 EGV den europäischen Regeln fast systematisch entzogen. Dies hat dazu geführt, dass immer noch nationale Einzelmärkte bestehen, was für die europäischen Unternehmen von Nachteil ist. Die Mitgliedstaaten ignorieren die von ihnen selbst beschlos-

3 Verhaltenskodex der EU-Mitgliedstaaten, die an der Europäischen Verteidigungsagentur (EVA) teilnehmen, über die Beschaffung im Bereich der Verteidigung; angenommen am 21. November 2005 durch die damals 24 Mitglieder der EVA (Dänemark ist nicht Mitglied).

4 Mitteilung der Kommission zu Auslegungsfragen bezüglich der Anwendung des Art. 296 EGV auf die Beschaffung von Verteidigungsgütern, 07.12.2006, KOM(2006) 779 endgültig.

5 Die Richtlinie befindet sich noch im Entstehungsstadium, wurde aber in den bisherigen Mitteilungen der Kommission zum Themenkomplex an verschiedenen Stellen angekündigt.

6 Vgl. Art. 10 der Richtlinie 2004/18/EG des Europäischen Parlamentes und des Rates vom 31. März 2004 über die Koordinierung der Verfahren zur Vergabe öffentlicher Bauaufträge, Lieferaufträge und Dienstleistungsaufträge. 
senen Gemeinschaftsregeln für fairen Wettbewerb, Transparenz und gegen Diskriminierung $^{7}$ und haben so die alleinige Entscheidungsgewalt im Bereich der Verteidigungsgüterpolitik. Dadurch ist es ihnen auch möglich ihre nationalen Rüstungsunternehmen zu bevorzugen. ${ }^{8}$ All dies führt jedoch dazu, dass Industrieinfrastrukturen innerhalb der Europäischen Union dupliziert wurden. Außerdem kann man feststellen, dass bisherige zwischenstaatliche Initiativen, auch die im Rahmen der zweiten Säule der Union, nicht dazu geführt haben einen europäischen Verteidigungsgütermarkt zu schaffen. Ein solcher Markt ist jedoch notwendig. Zum einen, um die Fortentwicklung und die Wettbewerbsfähigkeit der industriellen und technologischen Grundlage des Verteidigungssektors zu gewährleisten. Zum anderen könnte man damit das Kosten-Nutzenverhältnis ${ }^{9}$ der Ausgaben der Mitgliedstaaten optimieren und militärische Kapazitäten besser koordinieren, was im Rahmen der ESVP wichtig ist. Bis heute fehlt den Mitgliedstaaten der nötige Wille, um die erforderlichen Maßnahmen durchzuführen, die für die Bildung eines gemeinsamen Verteidigungsgütermarktes notwendig sind.

Die Europäische Kommission hat sich auf ihre Kompetenzen im Bereich des Gemeinsamen Markts gestützt, um für die Schaffung eines Europäischen Marktes für Verteidigungsgüter (kurz: EDEM, vom Englischen: European Defence Equipment Market) ihren Beitrag zu leisten. So hat sie bereits Ende der 80er Jahre festgestellt, dass bestimmte Teile der Verteidigungsgüterindustrie vollständig den europäischen Wettbewerbsregeln unterstellt werden können. Diesbezüglich wurden auch mehrere Studien in Auftrag gegeben. Aber erst eine im Jahr 2003 veröffentlichte Mitteilung ${ }^{10}$ wurde zu einem ersten wirklichen Schritt in diesem Zusammenhang. Das Grünbuch über Beschaffungen im Verteidigungssektor ${ }^{11}$, das im darauf folgenden Jahr veröffentlicht wurde, hat einen Denkprozess eingeleitet, mittels dessen Einsicht ermöglicht wird die öffentlichen Verteidigungsmärkte innerhalb der Europäischen Union zu öffnen und die Entwicklung des Wettbewerbs auf diesem Sektor zu befördern, ohne dabei die Besonderheiten dieses Gebiets auszublenden.

Die Vorschläge sind daran orientiert die Aufrechterhaltung der industriellen und technologischen Grundlagen zu sichern, die , Versorgungssicherheit ${ }^{6} \mathrm{zu}$ garantieren und die Staatsausgaben im Bereich der öffentlichen Verteidigung effektiver zu nutzen.

Bevor die verschiedenen Prozesse zur Harmonisierung des Verteidigungsgütermarktes innerhalb der Europäischen Union abgeschlossen werden können, muss die Kommission vorab die Anwendungsgrenzen des Art. 296 EGV präzise abstecken. Nur dadurch kann die Anzahl der Umgehungstatbestände verringert werden. Das Eingreifen zielt darauf ab, die missbräuchliche Nutzung dieser Ausnahmeregelung zu beenden und die nationalen Verteidigungsmärkte für den Wettbewerb zu öffnen. Missbrauch findet dadurch statt, dass Art. 296 EGV für Anwendungsfälle herangezogen wird, die nicht wirklich die , wesentlichen Sicherheitsinteressen ‘ eines Mitgliedstaates betreffen. Um die den Art. 296 EGV betreffenden

7 Heutzutage erfolgen in den Staaten der EU mehr als 50 Prozent der Verteidigungsbeschaffungen unter Umgehung des Gemeinschaftsrechts.

8 Die industriellen Kräfte der EU im Verteidigungssektor konzentrieren sich vor allem in Deutschland, Frankreich, Großbritannien, Italien, Spanien und Schweden, wo 85 Prozent der Ausgaben getätigt werden und sich 90 Prozent der Produktionskapazitäten befinden.

9 Zum Vergleich: die zusammengerechneten Ausgaben der Mitgliedstaaten der EU betragen 169 Milliarden Euro, was etwa der Hälfte der Ausgaben der USA entspricht; dennoch haben die EU-Mitgliedstaaten nur ein Fünftel der Verteidigungskapazitäten der USA. Vgl. Französische Kommission für Verteidigung und Streitkräfte: Bericht Nr. 42, 27.09.2005.

10 Europäische Kommission: Auf dem Weg zu einer Verteidigungsgüterpolitik der Europäischen Union, 11.03.2003, $\mathrm{KOM}(2003) 113$ endgültig.

11 Europäische Kommission: Grünbuch der Kommission über die Besaffung von Verteidigungsgütern, 23.09.2004, $\mathrm{KOM}(2004) 608$ endgültig. 
Ausnahmen zu reduzieren, hat die Kommission die oben genannte Mitteilung zu Auslegungsfragen bezüglich der Anwendung des Art. 296 EGV auf die Beschaffung von Verteidigungsgütern veröffentlicht (im Folgenden als , auslegende Mitteilung ' bezeichnet). Eine solche Mitteilung hatte sie bereits in ihrem Grünbuch über Beschaffungen im Verteidigungssektor 2004 angeregt. Diese jüngste Mitteilung soll sowohl Klarheit schaffen über das geltende Recht als auch über die Auslegung des Artikels. Auf dieser Grundlage können die Mitgliedstaaten nunmehr leichter unterscheiden zwischen den Fällen, die den europäischen Markt- und Vergaberegeln unterliegen, und den Ausnahmefällen.

Somit wird der Teil des europäischen Verteidigungsgütermarktes, welcher von den Ausnahmemöglichkeiten des Art. 296 EGV betroffen ist, nicht mehr willkürlich von den einzelnen Staaten definiert werden können. Die Reduzierung der Ausnahmefälle und die Anwendung einheitlicher Regeln, die dem Gemeinschaftsrecht entspringen, müssten die Öffnung des Verteidigungsgütermarktes für den europäischen Wettbewerb ermöglichen. Die Kommission versucht demzufolge einen bedeutenden Teil des europäischen Verteidigungsgütermarktes gemäß Art. 296 EGV zu ,vergemeinschaften', das heißt den europäischen Regeln zu unterstellen, um die Abweichung von der Regel wirklich zur Ausnahme werden zu lassen und nicht als Regel zu akzeptieren.

\section{,Erhellung der Grauzone' des Art. 296 EGV durch die auslegende Mitteilung der Kommission}

Eine der Hauptschwierigkeiten bei der Begrenzung der übermäßigen Anwendung der Ausnahmeregelung des Art. 296 EGV durch die Mitgliedstaaten besteht in der Ungenauigkeit des Begriffs der, wesentlichen Sicherheitsinteressen'. Die Kommission kann keine objektive Definition dieses sehr politischen Begriffs liefern. Dennoch wird sie zu einer restriktiven Auslegung beitragen, indem sie die Grundsätze präzisiert, auf deren Basis die Mitgliedstaaten in Übereinstimmung mit dem Gemeinschaftsrecht entscheiden müssen, ob der Anwendungsbereich der Ausnahmen erfüllt ist. Dabei stützt sie sich einerseits auf den Artikel selbst, der eine Verknüpfung des Objekts der Ausnahmeregelung mit den , wesentlichen Sicherheitsinteressen' eines Staates erfordert. Andererseits orientiert sie sich an der Rechtsprechung des Europäischen Gerichtshofs (EuGH).

Der Rechtssprechung zufolge sind die in einigen Artikeln des EG-Vertrags vorgesehenen Ausnahmefälle, so auch bei Art. 296, nicht für eine extensive Auslegung geeignet und lassen keinen immanenten Vorbehalt zu. ${ }^{12}$ Im Gegenteil, diese Ausnahmen müssen für jeden einzelnen Fall gerechtfertigt sein und ,[d] aher muß der Mitgliedstaat, der diese Ausnahmen in Anspruch nehmen möchte, nachweisen, daß die betreffenden Befreiungen nicht die Grenzen der genannten Tatbestände überschreiten"13.

\section{Kriterien für die Ausnahmeregelung des Art. 296 EGV}

In der am 6. Dezember 2005 veröffentlichten Mitteilung zu den Ergebnissen der Konsultation durch das Grünbuch $2004^{14}$ werden bereits einige generelle Aspekte vorweggenommen. So wird klargestellt, dass die Bedeutung von Verteidigungsgütern für die Sicherheits-

12 EuGH, Urteil vom 15.05.1986, Rs. 222/84 - Marguerite Johnston/Chief Constable of the Royal Ulster Constabulary, Slg. 1986, 1651, Randnr. 26.

13 EuGH, Urteil vom 16.09.1999, Rs. C-414/97 - Kommission der Europäischen Gemeinschaften/Königreich Spanien, Slg. 1999, I-05585, Randnr. 22.

14 Mitteilung der Kommission über die Ergebnisse der Konsultation zum Grünbuch über die Beschaffung von Verteidigungsgütern und über künftige Initiativen der Kommission, 06.12.2005, $\mathrm{KOM(2005)} 626$ endgültig. 
interessen der Mitgliedstaaten ,je nach politischen und militärischen Gegebenheiten sehr unterschiedlich sein“ kann. Dennoch ließe sich allgemein feststellen, dass sie ,in der Regel [...] mit der technischen Komplexität und der strategischen Wichtigkeit [steigt]“. ${ }^{15}$ Kurz gesagt: je komplexer ein militärisches Gut ist, desto sicherheitsrelevanter ist es. Daher wird wohl nur eine begrenzte Anzahl von hochsensiblen Verteidigungsgütern mit strategischer Funktion im Zuge der Anschaffung dieser Ausnahmeregelung unterliegen können.

Im Jahre 1958 hatte der Rat gemäß Art. 296 II EGV eine Liste ausgearbeitet, die fünfzehn Waffengattungen, Munitionsarten und Kriegsmaterialien anführt, die in die Ausnahmeregelungen einbezogen waren. Dabei muss aber berücksichtigt werden, dass die Liste keine zwingenden Rechtswirkungen entfaltet, da sie nicht formal veröffentlicht wurde und ihr Inhalt zum Teil veraltet ist. ${ }^{16}$ Daher wollte die Kommission diese Liste zunächst nicht in das Grünbuch aus dem Jahr 2004 einbeziehen. ${ }^{17}$ Sie wurde dann aber schließlich doch noch punktuell in die Mitteilung vom Dezember 2005 aufgenommen. Selbst wenn die Kommission immer noch die zu allgemein gehaltenen Formulierungen und damit die Ungenauigkeit der Liste aus dem Jahr 1958 betont, greift sie darauf zurück, um einige ihrer Schlussfolgerungen zum Anwendungsbereich des Art. 296 EGV zu bestärken in Bezug auf die Befreiungsberechtigung besonders sicherheitssensitiver Güterarten von den Gemeinschaftsvorschriften. ${ }^{18}$ Auf der anderen Seite leitet sie daraus ab, dass Güter, die von den Verteidigungsministerien nicht für spezielle militärische Zwecke bestimmt sind, dieser Ausnahme nicht unterliegen können. Tatsächlich zielten weder der Art. 296 EGV noch die Liste aus dem Jahr 1958 in ihrer Intention darauf ab, das Gemeinschaftsrecht zu unterlaufen. ${ }^{19}$ Die Kommission geht der EuGH-Rechtsprechung folgend also davon aus, dass alle neuen Ausstattungsgegenstände, die nicht ausdrücklich für militärische Zwecke vorgesehen sind und daher auch nicht in die Liste aus dem Jahr 1958 einbezogen wurden, nicht unter die Ausnahmeregelung des Art. 296 EGV fallen. ${ }^{20}$ Dies gilt auch dann, wenn sie möglicherweise zu einem späteren Zeitpunkt von den Mitgliedstaaten als ,,analog unter die Liste gehörend“ eingestuft worden waren. Dieser ergänzende Rahmen zum Begriff der ,wesentlichen Sicherheitsinteressen “ verringert die Grauzone des Art. 296 EGV deutlich.

Die auslegende Mitteilung selbst mag vielleicht diejenigen Kreise enttäuschen, die sich davon eine präzise Abgrenzung anhand bestimmter konkreter Kriterien oder Güterkategorien erhofft haben. Denn die Kommission stellt fest, dass die Mitteilung „,weder eine Interpretation der wesentlichen Sicherheitsinteressen der Mitgliedstaaten geben, noch im Voraus bestimmen [kann], auf welche Beschaffungsaufträge die Ausnahmeregelung des Artikels 296 EGV anwendbar ist und auf welche nicht“. ${ }^{21}$ Die Mitteilung ,,soll vielmehr den öffentlichen Auftraggebern Leitlinien zur Bewertung geben, ob die Inanspruchnahme der Ausnahmeregel gerechtfertigt ist“, denn es sei das „Vorrecht der Mitgliedstaaten, ihre wesentlichen Sicherheitsinteressen zu definieren, und ihre Verpflichtung, diese zu schützen“. Schließlich

15 Ebenda, Punkt I.2.

16 Machen Revolver, Pistolen, militärische Panzerreparaturfahrzeuge, etc. immer noch die , wesentlichen Sicherheitsinteressen' eines Mitgliedstaats aus?

17 KOM(2004)608, Punkt I.3.1

$18 \operatorname{KOM}(2005) 626$, Punkt I.3.

19 Art. 296 Abs. 1 b) EGV regelt vielmehr, dass Maßnahmen zum Schutz der wesentlichen Sicherheitsinteressen eines Mitgliedsstaats in Abweichung von den Regeln des Gemeinschaftsrecht die Wettbewerbsbedingungen auf dem Gemeinsamen Markt hinsichtlich der nicht eigens für militärische Zwecke bestimmten Waren nicht beeinträchtigen dürfen.

20 EuGH, Urteil vom 30. September 2003, Rechtssache T-26/01, Fiocchi Munizioni/Kommission, Slg. 2003, II3951, Randnr. 61.

$21 \operatorname{KOM}(2006) 779$ endgültig, Einleitung S. 3. 
erlaubt Art. 295 I b) EGV den Mitgliedstaaten Maßnahmen zu treffen, die „,eines Erachtens für die Wahrung seiner wesentlichen Sicherheitsinteressen" erforderlich sind. Jedoch müssen die Mitgliedstaaten dabei „mit großer Sorgfalt jeden Beschaffungsvorgang einzeln daraufhin prüfen, ob die Nichtanwendung der Gemeinschaftsregeln gerechtfertigt ist oder nicht". ${ }^{22}$ Der mitgliedstaatliche Ermessensspielraum bei Bewertung der wesentlichen Sicherheitsinteressen ist nicht unbegrenzt. Die „Kommission kann den EuGH auch unmittelbar anrufen, wenn sie der Auffassung ist, dass ein Mitgliedstaat die in Artikel 296 EGV vorgesehenen Befugnisse missbraucht. In diesem Fall liegt die Beweislast, dass die Inanspruchnahme der Ausnahmeregel gerechtfertigt ist, beim betroffenen Mitgliedstaat. “23 Diese zurückhaltende Gestaltung des Rahmens für die Anwendung des Art. 296 EGV dürfte darauf zurückzuführen sein, dass sich die Frage zu einem zentralen Blockadepunkt für die Mitgliedstaaten entwickelt hat. ${ }^{24}$

\section{Der Begriff der ,wesentlichen Sicherheitsinteressen " und der zwischenstaatliche Verhal- tenskodex}

Die Bereiche, die heute wohl als ,wesentliche Sicherheitsinteressen' bezeichnet werden können, müssen in einer engeren Auslegung ermittelt werden als dies im Rahmen der Liste aus dem Jahr 1958 der Fall war. Denn diese wurde in einem ganz anderen geopolitischen Kontext verfasst. ${ }^{25}$ Wie es der Bericht des Europäischen Parlaments in Bezug auf das Grünbuch unterstreicht, hält es ,,angesichts der ohnehin bestehenden gegenseitigen Abhängigkeit der Mitgliedstaaten in Bereichen wie etwa Währung oder Energie eine restriktive Interpretation der nationalen Sicherheitsinteressen für angebracht" und es wird an gleicher Stelle die Frage aufgeworfen, in welchem Umfang heute überhaupt noch sinnvoll nationale von gemeinsamen europäischen Sicherheitsinteressen abgegrenzt werden können“". ${ }^{26}$ Auch der zwischenstaatliche Verhaltenskodex für die Beschaffung von Verteidigungsgütern vom 21. November 2005 gibt in dieser Auslegungsfrage keine näheren Abgrenzungskriterien oder Definitionen. Der Kodex findet Anwendung in den Bereichen, für die der Ausnahmetatbestand des Art. 296 EGV von den Mitgliedstaaten in Anspruch genommen wird. Jedoch werden von diesem Kodex wiederum einige Segmente des Marktes ausgenommen: der Erwerb von nuklearen Waffen und Antriebssystemen sowie von Gütern und Dienstleistungen im gesamten Bereich der ABCKriegsführung und der Kryptografie. Ferner fallen auch Beschaffungen im Bereich Forschung und Technologie (F\&T) sowie die innerhalb einer Kooperation getätigten Käufe unter die Herausnahme aus dem Anwendungsbereich. Für die Mitgliedstaaten der EVA gehören diese Marktsegmente offensichtlich zu den grundlegendsten ihrer, wesentlichen Sicherheitsinteressen'. Der ausdrückliche Ausschluss dieser Bereiche vom Anwendungsbereich der durch den Kodex formulierten Prinzipien unterstreicht, dass die Mitgliedstaaten sie als äußerst ,sensibel ${ }^{\text {‘ }}$ und von besonderer strategischer Bedeutung betrachten. Selbst wenn die Klarstellung sich auf Bereiche beschränkt, die aus gutem Grund zu den ,wesentlichen Sicherheitsinteressen' der

$22 \mathrm{KOM}(2006) 779$ endgültig, Punkt 5.

$23 \operatorname{KOM}(2006) 779$ endgültig, Punkt 6.

24 So stellt z.B. Großbritannien in seiner Stellungnahme zum Grünbuch vom 15. Februar fest, dass es sich jedweder Regelung widersetzen werde, die die Rechte aus Art. 296 EGV verletzen oder zu regeln versuchen sollte, wenn dabei die Angelegenheiten im Kompetenzbereich der Mitgliedstaaten betroffen sind. Die Haltung Frankreichs geht in eine ähnliche Richtung.

25 Zum einen durch den internationalen Kontext des Kalten Kriegs, zum anderen durch die wesentliche geringere Zahl der Mitgliedstaaten. Außerdem war man zum damaligen Zeitpunkt noch weit von einem , politischen Europa' und einer GASP entfernt.

26 Bericht des Europäischen Parlamentes, Ausschuss für Binnenmarkt und Verbraucherschutz, zum Grünbuch über die Beschaffung von Verteidigungsgütern vom 10.10.2005, A6-0288/2005, Punkt 12. 
Staaten zählen - zumindest, was den ersten Teil der oben genannten Liste betrifft - wird im Umkehrschluss die ,Grauzone' des Art. 296 EGV deutlich. ${ }^{27}$

Da die Breite des Anwendungsbereichs des Artikels nicht detailliert bestimmt ist, kann der Verhaltenskodex auf einen großen Teil des Verteidigungsgütermarktes angewandt werden. Denn je weiter die Mitgliedstaaten die Ausnahmeregelung auslegen und dabei dem supranationalen Gemeinschaftsrecht eine Absage zu erteilen, desto mehr findet der zwischenstaatliche Kodex Anwendung. Allerdings stimmen die zwischenstaatlichen Ansätze auf der einen Seite und die der Kommission im Rahmen der Bildung eines europäischen Verteidigungsgütermarktes auf der anderen in ihren Grundintentionen weitgehend überein. Dies erklärt, warum trotz der unterschiedlichen Ansichten der Mitgliedstaaten bezüglich der Schwerpunktlegung zwischen den beiden Wegen dennoch ein weitgehender Einklang zu beobachten ist. ${ }^{28}$ Dennoch ist zu vermuten, dass einige der Staaten sich erhoffen mit einer ins eigene Ermessen gelegten Definition ihrer, wesentlichen Sicherheitsinteressen " weiterhin Einfluss auf den Marktöffnungsprozess zu behalten.

Was bei der Anwendung von Art. 296 EGV Schwierigkeiten aufwirft, ist die Einordnung des Erwerbs von Waren und Dienstleistungen, ,bei denen es sich zwar um Verteidigungsgüter handelt, die aber nicht (zwangsläufig) von wesentlicher Bedeutung für die Wahrung ihrer Sicherheitsinteressen [der Mitgliedstaaten] sind“29. Bei Verteidigungsgütern sollte also einzig die strategische Bedeutung der Ausrüstung das entscheidende Kriterium darstellen, ob ein Marktsegment der Ausnahmeregelung unterliegt. Aber dieser Begriff der ,strategischen Bedeutung' ist immer noch breit genug, um den Mitgliedstaaten einen gewissen Ermessensspielraum zu überlassen, selbst wenn er weniger breit ist als der Begriff der, wesentlichen Sicherheitsinteressen".

Auch im Hinblick auf die geltenden EU-Regelungen im Bereich der Vergabe ist schon heute erkennbar, dass die auslegende Mitteilung mit ihren Rahmen für die Anwendung des Art. 296 EGV nicht ausreicht. Somit ist allein auf dieser Grundlage eine Öffnung der Verteidigungsmärkte nicht zu erwarten. ${ }^{30}$ Genau wie die große Mehrheit der Akteure, die in der Konsultation zum Grünbuch Stellung genommen haben, teilt die Kommission die Einschätzung, ,dass Art. 296 EGV oft falsch interpretiert wird, und [...] die geltenden Vergaberichtlinien sich trotz der kürzlich erfolgten Anpassungen in vielen Fällen nicht für Wehrbeschaffungen eigenen. “31 Es gilt daher den möglichen Inhalt einer neuen Richtlinie zu erörtern, die

27 Dagegen könnten es bei den Beschaffungen im Bereich Forschung und Technologie (F\&T) sowie den innerhalb einer Kooperation getätigten Käufen künftig in Frage stehen, ob sie zu den , wesentlichen Sicherheitsinteressen ' gehören. Dem deutschen Vorschlag aus der Antwort zum Grünbuch aus dem Jahr 2004 folgend, könnte z.B. beschlossen werden, dass Forschungs-, Technologie- und Entwicklungsprogramme künftig in das Forschungsrahmenprogramm der EU integriert werden.

28 In den Stellungnahmen der Mitgliedstaaten zum Grünbuch der Kommission aus dem Jahr 2004 wurde die Bedeutung des Verhaltenskodexes gegenüber der Kommissionstätigkeit im Bereich des europäischen Verteidigungsgütermarktes häufig betont. Während Frankreich schätzt, dass eine auslegende Mitteilung keine neuen Inhalte bringen dürfte (JMD/jf/544 MICA/182/2005 vom 25.02.2005), stellt sich Großbritannien nicht gegen das ihr innewohnende Grundprinzip. Beide Staaten sind sich wiederum darin einig, dass der Verhaltenskodex eine wesentliche Rolle bei der Bildung eines europäischen Verteidigungsgütermarktes spielen könnte. Deutschland begrüßt in ihrem „Aktionsprogramm für den Rüstungsmarkt“ eine auslegende Mitteilung, unterstreicht aber auch gleichzeitig die Notwendigkeit sich im Vornherein klar zu werden über die notwendigen Maßnahmen zur Entwicklung und Regelung eines europäischen Verteidigungsgütermarktes.

$29 \operatorname{KOM}(2005) 626$, Punkt I.3.

30 „Eine Mitteilung zu Auslegungsfragen würde die Zurückhaltung der Mitgliedstaaten in Bezug auf die Anwendung der geltenden Vergaberichtlinien auf Wehrbeschaffungen nicht ausräumen. Ihre transparenzschaffende und wettbewerbssteigernde Wirkung wäre daher weitgehend auf Güter beschränkt, bei denen es sich nicht um Kriegsmaterial handelt.“, KOM(2005)626, Punkt II.2.1.(2).

$31 \operatorname{KOM}(2005) 626$, Punkt II.2. 
im kommenden Jahr vorgeschlagen werden sollte. Dazu wäre es zunächst einmal notwendig in einer Studie zu untersuchen auf welche Weise die im zwischenstaatlichen Verhaltenskodex aus dem Jahr 2005 festgelegten Regeln an die Verteidigungsmärkte, die dem Art. 296 EGV unterliegen, angepasst worden sind.

\section{Erfolgsbedingungen zur Liberalisierung des europäischen Verteidigungsgütermarktes}

Nach der ,nicht legislativen“ Etappe der auslegenden Mitteilung zum Art. 296 EGV will die Kommission eine Richtlinie über die Verteidigungsmärkte vorlegen. Damit würde die Liberalisierung des Verteidigungsgütermarktes einen wesentlichen Schritt weiterkommen. In der Tat müsste man so die Vergabeverfahren auf den Verteidigungsmärkten der Europäischen Union koordinieren. So könnte das Vergaberecht den Anforderungen im spezifischen Sektor der Verteidigung angepasst werden. Eine Richtlinie stellt das wohl wirksamste Mittel dar, um den Markt zu liberalisieren und den europäischen Wettbewerb zu stärken. Außerdem enthält der im Jahr 2005 angenommene zwischenstaatliche Verhaltenskodex ebenfalls Regeln zur Verbesserung der Transparenz und zur Erleichterung des Wettbewerbs. Folglich laufen beide Initiativen - so sie auch erfolgreich umgesetzt werden ${ }^{32}$ - darauf hinaus, eine gewisse Liberalisierung des Verteidigungsgütermarktes zu fördern. Dabei soll gleichwohl den Eigenheiten des Verteidigungsgütersektors Rechnung getragen werden. Daher stellt sich nun die Aufgabe, die Natur der in diesem Zusammenhang geplanten Regeln Schritt für Schritt zu analysieren.

\section{Die Richtlinie zur Liberalisierung der Verteidigungsgütermärkte}

Die Kommission schätzt, dass die Verteidigungsgütermärkte nur dann liberalisiert werden können und ein ausreichendes Wettbewerbsniveau auf europäischer Ebene nur dann vollständig zu erreichen ist, wenn die Verfahrensregeln zum Abschluss von Verträgen zur Beschaffung von Gütern (Waffen, Munition und Kriegsmaterial) sowie von Dienst- und Werkleistungen in diesem Sektor auf EU-Ebene einheitlich geregelt werden. ${ }^{33}$ Sie rechtfertigt ihren Vorschlag mit der Möglichkeit, die Fragmentierung der Nachfrage in der EU (27 nationale Käufer) aufzuheben und das geltende Regelwerk (27 verschiedene Rechtsrahmen und Verfahrensregeln) ${ }^{34}$ zusammenzuführen. Um die Herstellung eines einheitlicheren Regelwerkes innerhalb der Europäischen Union zu erreichen und die Erstellung eines europäischen Verteidigungsgütermarktes zu begünstigen, beabsichtigt die Kommission die Ausarbeitung einer Sektoren-Richtlinie. Dabei folgt sie dem Prinzip, das sie für die Öffnung der Wasser-, Energie- und Transportmärkte in den 1990er Jahren bereits angewandt hat. Die europäischen Vergaberegeln würden somit den speziellen Bedürfnissen des Verteidigungssektors angepasst. Durch eine solche Richtlinie wird nach Ansicht der Kommission die Anwendung der europäischen Regeln auf die Verteidigungsgütermärkte erleichtert.

32 Viele Mitgliedstaaten sind einer solchen Richtlinie noch abgeneigt. Der Verhaltenskodex dagegen trat zum 1. Juli 2006 in Kraft und gilt für alle EVA-Mitgliedstaaten, außer Spanien und Ungarn, die ihm vorerst noch nicht beitreten möchten. Jedoch haben diese beiden Länder auf die Möglichkeit eines späteren Beitritts hingewiesen.

33 Eine solche Richtlinie würde nur diejenigen Verträge erfassen, welche aufgrund der Natur der Sache (ratione materiae) unter den Anwendungsbereich des Art. 296 EGV subsumiert werden, aber für die das Berufen auf diese Vorschrift nicht gerechtfertigt ist oder nicht geltend gemacht worden ist. Somit unterliegt der Markt für Güter, die nicht für spezifisch militärische Zwecke vorgesehen sind, weiterhin der Richtlinie 2004/18/EG. Die Marktsegmente, bei denen sich Mitgliedstaaten zurecht auf Art. 296 EGV berufen, werden durch den zwischenstaatlichen Verhaltenskodex geregelt.

34 Die Kommission folgert in ihrer Mitteilung aus dem Jahr 2005: „Es herrscht generell Einigkeit darüber, dass die unterschiedlichen einzelstaatlichen Vergabevorschriften und ihre praktische Anwendung Transparenz und Wettbewerb auf den Verteidigungsmärkten beeinträchtigen."Vgl. KOM(2005) 626, Punkt I.3. 
Die Vereinheitlichung der Vergabe im Verteidigungsbereich und ihre Anpassung an diesen Marktsektor erfordern in erster Linie eine verbesserte Informationsgewinnung über die nationalen Ausschreibungen innerhalb der Europäischen Union. Tatsächlich kann echter Wettbewerb und eine wirkliche Öffnung der Verteidigungsgütermärkte nur zustande kommen, wenn jedes europäische Unternehmen an einer Ausschreibung in einem anderen Mitgliedstaat unter denselben Bedingungen teilnehmen kann wie die nationalen Unternehmen. Die Mitteilung der Kommission vom Dezember 2005 mit den Ergebnissen der Konsultation zum Grünbuch betont, dass die Mehrheit der Antwortenden eine solche Richtlinie für nützlich halte, um damit unter anderem ,ein geeignetes zentralisiertes System für die Bekanntmachung" zu ermöglichen. ${ }^{35}$

In zweiter Linie impliziert die Schaffung eines wettbewerbsorientierteren und homogeneren europäischen Verteidigungsgütermarktes, dass die Mitgliedstaaten die Einhaltung europäischer Prinzipien wie Gleichbehandlung und Transparenz bei der Vergabe garantieren. Gleichzeitig sollte diese Vereinheitlichung der Verfahrensregeln zu mehr Praktikabilität in der Anwendung führen und den Besonderheiten des Verteidigungssektors genügen. Dementsprechend stellt die Kommission fest, dass die Mehrheit der Rückmeldungen zum Grünbuch die Einführung des Verhandlungsverfahrens mit vorangegangener öffentlicher Aufforderung zur Teilnahme wünscht. ${ }^{36} \mathrm{Um}$,eine potenzielle Diskriminierung der Lieferanten zu vermeiden“, schlug das Grünbuch vor, dass der Auftragsgegenstand in der Bekanntmachung ,anhand technischer Leistungsparameter beschrieben werden“ könne. ${ }^{37}$ Damit aber die Richtlinie gleichfalls besonderen Situationen, wie zum Beispiel militärischen Notfällen, gerecht werden kann, müsste sie außerdem vorsehen, dass Vertragsabschlüsse in solchen Sonderfällen auch ohne vorangehende öffentliche Ausschreibung im Verhandlungsverfahren durchgeführt werden dürfen. Ebenso ist es wichtig, dass neue Auswahlkriterien den besonderen Erfordernissen von Beschaffungsverträgen im Verteidigungssektor (wie beispielsweise Vertraulichkeit, Versorgungssicherheit und Wahrung des Verteidigungsgeheimnisses) gerecht werden. ${ }^{38}$ Aus der Mehrheit der Antworten auf das Grünbuch ist zu entnehmen, dass die Richtlinie ein ausreichendes Wettbewerbsniveau für die ganze Versorgungskette sicherstellen muss. Es sind also alle Phasen von der Entwicklung über die Fertigung bis hin zur Wartung des militärischen Materials zu berücksichtigen. Die Vielzahl der in den Bereitstellungsprozess involvierten Wirtschaftsakteure, von denen viele kleine und mittlere Unternehmen (KMU) sind, machen dessen Komplexität aus. Einheitliche Vergaberegeln könnten auch das Problem der Kompensationsgeschäfte (Offsets) lösen. Das ist besonders wichtig, denn diese Praxis verursacht - genau wie die Politik des , juste retour " - große Wettbewerbsverzerrungen und künstliche Arbeitsteilungen zwischen Industriepartnern, was wiederum die Effizienz der öffentlichen Märkte behindert. ${ }^{39}$

Die neue Richtlinie würde der Nichtanwendung der Vergaberichtlinie 2004/18/EG auf den Verteidigungsgütermärkten eine sinnvolle Lösung entgegensetzen. Die verbesserte Praktikabilität durch die neue Richtlinie würde eine wirkliche Alternative zu den nationalen Verfahren eröffnen und könnte folglich zugunsten des Gemeinschaftsrechts die Häufigkeit

$35 \operatorname{KOM}(2005) 626$, Punkt II.2.2.(1).

36 Diese Vorgehensweise würde das traditionelle offene Verfahren der Aufforderung zur Einreichung von Angeboten im Amtsblatt der Europäischen Union ersetzen, denn dieses ist mit den Anforderungen an die Vertraulichkeit im Rüstungssektor wenig vereinbar.

$37 \mathrm{KOM}(2004) 608$, Punkt II.2.2.

38 Denn im Verteidigungssektor können die Auswahlkriterien nicht allein auf technischen, wirtschaftlichen und finanziellen Gesichtspunkten aufbauen, wie dies in den allgemeinen Vergaberichtlinien der Fall ist.

39 Bericht des Europäischen Parlamentes, Ausschuss für Binnenmarkt und Verbraucherschutz, zum Grünbuch über die Beschaffung von Verteidigungsgütern vom 10.10.2005, A6-0288/2005, Punkt 4. 
der Anwendung des Ausnahmetatbestands reduzieren. Auf der anderen Seite könnte die Richtlinie ohne die Berufung auf Art. 296 EGV vorwegzunehmen ,die Fälle präzisieren, in denen die Voraussetzungen für die Inanspruchnahme der Ausnahmeregelung eindeutig und zweifelsfrei erfüllt sind (wie zum Beispiel bei nuklearem Material). “40

Aus all diesen Betrachtungen wird ersichtlich, dass die Richtlinie überall dort einen europäischen Verteidigungsgütermarkt schaffen könnte, wo Art. 296 EGV keine Anwendung findet. Denn die Einführung von einheitlichen Vergaberegeln auf EU-Ebene würde sicherlich die nötigen Bedingungen für eine aktive Öffnung dieses Marktes schaffen und die Entstehung eines europäischen Verteidigungsgütermarktes begünstigen. Außerdem würde der verpflichtende Charakter einer Richtlinie dazu beitragen, dass der Prozess effektiv umgesetzt wird. Auch wenn Wettbewerb und Transparenz untrennbar mit einer Öffnung verknüpft sind wird den Eigenheiten des Verteidigungsgütermarktes durch die Verankerung zusätzlicher Prinzipien Rechnung getragen. Damit wären die Ziele nicht ausschließlich wirtschaftlicher Art. Zwar würde einerseits angestrebt die Kosten der Militärausgaben zu senken und eine wirtschaftlichere Verwendung der öffentlichen Ausgaben zu erreichen. Andererseits würden aber auch industrielle und verteidigungspolitische Zwecke abgesichert wie zum Beispiel die Gewährleistung der Versorgungssicherheit und das Schließen der Lücken des europäischen Aktionsplans über die Kapazitäten. Jedoch bekennt die Kommission, dass eine Richtlinie das Problem der Abgrenzung des Anwendungsbereichs von Art. 296 EGV nicht lösen würde. ${ }^{41}$ Wie kann die Richtlinie also mit dem zwischenstaatlichen Verhaltenskodex, der ja genau diesen der Ausnahmeregelung des Art. 296 EGV unterliegenden Markt betrifft, verbunden werden und welche Auswirkungen könnte der Kodex zugunsten der Marktöffnung haben.

\section{Der zwischenstaatliche Verhaltenskodex: Vorbedingung oder Ergänzung zu einer Verteidigungsgüterrichtlinie?}

Eine Richtlinie findet derzeit noch nicht die Zustimmung aller Mitgliedstaaten. Unter den Gegnern sind einige der wichtigsten europäischen Produzenten und Abnehmer von Verteidigungsgütern. So hofft beispielsweise Frankreich, dass vor allem die Mitgliedstaaten einen europäischen Verteidigungsgütermarkt schaffen müssen. Der Richtlinie müsse eine vorherige Einigkeit zwischen den Mitgliedstaaten über ihre Beschaffungspolitik vorangehen. Dies könne nur über politische Maßnahmen erreicht werden wie den zwischenstaatlichen Verhaltenskodex für die Verteidigungsgütermärkte, der innerhalb der EVA ausgearbeitet wurde. ${ }^{42}$ Frankreich bevorzugt offensichtlich eine Konsolidierung des Marktes in einem zwischenstaatlichen Rahmen. Demnach wird die Ausarbeitung einer Richtlinie erst zu einem späteren Zeitpunkt als sinnvoll angesehen, nämlich dann, wenn der zwischenstaatliche Prozess das Entstehen eines europäischen Verteidigungsgütermarktes ausreichend vorangetrieben haben wird. Großbritannien seinerseits befürwortet eine wirtschaftlich und liberal ausgerichtete Vorgehensweise, um die grundlegende Rolle des Verhaltenskodexes bei der freiwilligen Öffnung des Marktes durch die Mitgliedstaaten zu untermauern. Dies schließe die Förderung des freien Wettbewerbs auf dem Markt durch die freiwilliger Einhaltung der Kodexregeln und die Anwendung des Prinzips der Gewinnmaximierung ein.

Im Hinblick darauf, dass sich Beschaffungspraktiken und -modalitäten in der Europäischen Union noch stark unterscheiden, halten es einige Mitgliedstaaten, so auch Deutsch-

40 KOM(2004)608, Punkt II.2.2. Außerdem könnten die Kriterien des Verhaltenskodexes der Kommission als Referenz dienen.

$41 \operatorname{KOM}(2005) 626$, Punkt II.2.2.(1).

42 Siehe die Stellungnahme Frankreichs zum Grünbuch vom 25.02.2005 (JMD/jf/544 MICA/182/2005). 
land, ,unter diesem Gesichtspunkt [für] ratsam, die Angleichung der Vergabepolitik der Mitgliedstaaten voranzutreiben, bevor man ihre Vergabevorschriften koordiniert. “43 Der Argumentation des Europäischen Parlaments folgend, kann man jedoch hiergegen einwenden, dass die europäischen Richtlinien zur Marktöffnung in anderen spezifischen Sektoren (zum Beispiel: Energie, Telekommunikation) beschlossen worden sind, obwohl die industrielle Lage von Mitgliedstaat zu Mitgliedstaat sehr unterschiedlich war. Dies kann auch den Akteuren entgegengehalten werden, die auf das Grünbuch geantwortet haben, dass eine Richtlinie nur im Falle eines Misserfolgs eine Alternative zum Kodex darstellen würde.

Der Verhaltenskodex gründet auf der Anerkennung durch die Mitgliedstaaten. Den Kern davon bilden fünf Prinzipien:

- freiwilliges beziehungsweise nicht verpflichtendes Vorgehen

- eine gerechte und gleiche Behandlung der Anbieter

- Transparenz und gegenseitige Rechenschaftspflicht

- wechselseitige Unterstützung

- wechselseitiger Nutzen.

Die Bildung eines Internetportals zur Veröffentlichung der Ausschreibungen könnte dabei behilflich sein diese Prinzipien sowie die Öffnung der Märkte zu verwirklichen, um die nötigen Bedingungen für einen wirklichen Wettbewerb und Transparenz zu schaffen. Auf dem Portal würden Beschaffungsabsichten im Wert von über einer Million Euro im Marktsegment, das von der Ausnahmeregelung des Art. 296 EGV profitiert, zugänglich gemacht (bis auf Ausnahmefälle). Die Idee für das Internetportal ist von der Kommission für den Teil des Markts aufgenommen worden, der nicht der Ausnahmeregel unterliegt und daher Teil ihres Zuständigkeitsbereichs ist. Der Verhaltenskodex legt den Schwerpunkt vor allem auf die freiwillige Entwicklung von guter Verwaltungspraxis beim Erwerb von Verteidigungsgütern. So geht der Kodex im Gegensatz zur Richtlinie eher in die Richtung die Rationalisierung des Markts und die Begründung eines Wettbewerbsregimes ohne eine Koordinierung der nationalen Rechtsvorschriften erreichen zu wollen. Die Prinzipien des Kodexes dürften dabei zu Wechselwirkungen zwischen den nationalen Regeln und dem Funktionieren des Verteidigungsgütermarktes führen, was wohl letztlich die Staaten in die Pflicht nehmen würde, das Verfahren und die Kriterien der Angebotsauswahl präzise offenzulegen. Diese Ziele sind mit denen der Kommission identisch, den erforderlichen Wettbewerb im System der Zulieferer- und Subunternehmerverträge sicher zu stellen.

Man kann schließlich feststellen, dass die vom Verhaltenskodex propagierten Prinzipien genau wie die Richtlinie auf eine erfolgreiche Liberalisierung des großen Teils des Verteidigungsgütermarktes abzielen. Die Rolle der Kommission und die Tätigkeit der Mitgliedstaaten über den Verhaltenskodex sind daher keine konkurrierenden Wege, sondern können vielmehr als sich ergänzende Prinzipien betrachtet werden. Dabei werden beide Mittel jeweils den speziellen Anforderungen des Teilbereichs des Marktes gerecht, für den sie Anwendung finden und laufen letztlich darauf hinaus Wettbewerb und Transparenz durch die Öffnung der Verteidigungsgütermärkte zu fördern. Die zwischenstaatlichen und europäischen Initiativen haben noch weitere gemeinsame Ziele: die Wahrung der industriellen und technologischen Grundlage sowie der Versorgungssicherheit und darüber hinaus die Bildung eines europäischen Verteidigungsgütermarktes. Nur die Wege zum Ziel unterscheiden sich.

Der Verhaltenskodex ermöglicht es den Mitgliedstaaten eine gewisse Kontrolle zu bewahren über Schnelligkeit und Anwendungsbreite der Marktöffnung hin zu einem Europäischen Verteidigungsgütermarkt. Es ist aber festzustellen, dass ein effizienter Liberalisie-

$43 \mathrm{KOM}(2005) 626$, Punkt II.3. 
rungsprozess mit staatlicher Kontrolle wenig kompatibel ist: die zwischenstaatlichen Vorläuferinitiativen auf diesem Gebiet (beispielsweise WEAG Western European Armaments Group, OCCAR Organisation Conjointe de Coopération en matière d'Armement) haben gezeigt, dass sich einige Staaten sehr viel Zeit nehmen, um die nicht rechtsverbindlichen Maßnahmen umzusetzen oder diese nur in geringstmöglichem Umfang anwenden. Trotzdem könnte sich aufgrund des Drucks durch die Verteidigungsgüterindustrie die Anwendung des im Rahmen der EVA ausgearbeiteten Kodexes als wirkungsvoll erweisen; auf der anderen Seite gibt es noch die Einflussnahme auf den Prozess durch die Kommission, um den Teil des Marktes zu regeln, der in ihren Anwendungsbereich fällt.

Eine Liberalisierung des europäischen Verteidigungsgütermarktes schließlich macht eine Verbesserung der Interoperabilität der nationalen Verteidigungsgüter erforderlich. Diese würde den europäischen Wettbewerb erleichtern und zu Synergien zwischen den nationalen Industrien führen. Zur Erhöhung der Interoperabilität von Verteidigungsgütern bedarf es der Entwicklung von (Referenz-)Normen auf europäischer Ebene. Die europäischen Normungsinstitute CEN, CENELEC und ETSI unterstützen die europäische Gesetzgebung bereits heute auf nicht-legislativer, freiwilliger Ebene auf Initiative der Industrien der Mitgliedstaaten, um durch den Abbau von technischen Handelshemmnissen den Binnenmarkt zu verwirklichen. Die Entwicklung von gemeinsamen europäischen Normen auch im Bereich der Verteidigungsgüter kann bestehende Barrieren in diesem Markt beseitigen und zur Schaffung grenzüberschreitenden Wettbewerbs beitragen. Die EVA muss weiterhin eine wichtige Rolle als Plattform zur Koordinierung nationaler Rüstungsaktivitäten und im Hinblick auf eine gemeinsame Normenpolitik spielen. Auch die Leistungsfähigkeit einer gemeinsamen, europäischen (Sicherheits- und) Verteidigungspolitik, heute unter anderem die Effektivität des Eurocorps, von EUROFOR und der schnellen Eingreiftruppe, hängt von der Interoperabilität europäischer Verteidigungsgüter ab.

\section{Schlussfolgerung}

Die Liberalisierung des europäischen Verteidigungsgütermarktes (Öffnung der nationalen Märkte für Wettbewerb und Transparenz) ist ein komplexer Prozess, in dem gleichzeitig die Verteidigungsgüterunternehmen, die Mitgliedstaaten und auf europäischer Ebene Kommission und Europäisches Parlament intervenieren. Diese Vielzahl an Akteuren erzeugt zwangsläufig Friktionen: einerseits zwischen wirtschaftlichen und politischen Ansätzen, und andererseits zwischen intergouvernementaler und gemeinschaftlicher Herangehensweise. Aber diese Friktionen können auch der Anfang einer die Liberalisierung des Markts vorantreibenden Dynamik sein. Ebenso könnte der Druck der Firmen, die sich in der Phase der Konsolidierung auf europäischer Ebene befinden und für die eine Marktöffnung wirtschaftlich notwendig ist, die Aktionen der Mitgliedstaaten beeinflussen. Vor dem Hintergrund all dieser aktiven Prozesse ist zu erwarten, dass die Umgehung des Gemeinschaftsrechts schrittweise ersetzt wird durch eine konsistente Anwendung der Ausnahmeregelung des Art. 296 EGV unter Beachtung der durch das Gemeinschaftsrecht gebotenen Ermessensgrenzen und der Rechtssprechung des EuGH. Diese Interaktionen müssten mittelfristig zur Entwicklung eines wirklichen europäischen Verteidigungsgütermarktes führen. Geprägt wäre dieser Markt durch die Angepasstheit der Wettbewerbs- und Transparenzregeln an die spezifischen Erfordernisse im Verteidigungssektor und durch die Unterhaltung einer industriellen und technologischen Grundlage der Verteidigung, die die Versorgungssicherheit garantiert. Auch wäre bei alldem eine wirtschaftlichere Verwendung der öffentlichen Investitionsausgaben sichergestellt. 
In dieser Hinsicht kann man den Prozess eine , gebändigte Liberalisierung ' nennen, insoweit als sie nicht nur auf den Gesetzen des Markts gründet. Es ist ersichtlich, dass die Liberalisierung des europäischen Verteidigungsgütermarktes schon begonnen hat. Die zunehmende Annäherung der Interessen wird dazu führen, dass letztendlich der gemeinschaftliche Ansatz bevorzugt wird. Nichtsdestotrotz muss noch unterstrichen werden, dass die Schaffung eines europäischen Verteidigungsgütermarktes neben der Marktliberalisierung noch weitere ergänzende Maßnahmen erfordert. Beispielhaft könnten hier die Harmonisierung der Exportpolitik, die Überprüfung der Beihilferegelungen, der Regeln bezüglich Transit und Transfer sowie der Rahmenbedingungen für Privatisierungen genannt werden.

Als letztes ist noch darauf hinzuweisen, dass eine weitere Liberalisierung des europäischen Verteidigungsgütermarktes auch eine soziale Dimension aufweist, deren Bedeutung nicht zu unterschätzen ist und gerade vor dem Hintergrund aktueller Entwicklungen Beachtung finden sollte. Denn die Entwicklung des Marktes hat Auswirkungen auf nationale Arbeitsmärkte: die Industrien sind bedeutende Arbeitgeber und die politisch Verantwortlichen sind besonders für diese Fragen sensibilisiert. Das Beispiel EADS hat dies verdeutlicht: die Krise hatte zwar ihren Beginn in internen Umstrukturierungsplänen von Airbus, besprochen und auch entschieden wurde sie jedoch auf höchster Ebene deutsch-französischer Politik. Ein zusätzlicher Indikator dafür, dass die weitere Entwicklung des europäischen Verteidigungsgütermarktes auch von den Interessen der nationalen politischen Akteure, gerade im Hinblick auf die Arbeitsmarktsituation und den sich hieraus generierenden gesellschaftlichen Erwartungsdruck, bestimmt werden wird.

\section{Verwaltungsreformen}

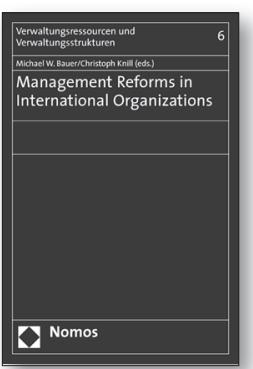

\section{Management Reforms in International Organizations}

Herausgegeben von Michael W. Bauer und

Christoph Knill

2007, ca. 226 S., brosch., ca. 39,- €, ISBN 978-3-8329-2572-7

(Verwaltungsressourcen und Verwaltungsstrukturen, $B d .6$ )

Erscheint Mai 2007

Erstmalig liefern die Autoren einen Ansatz, die Geschwindigkeit, die Akzeptanz sowie den Umfang von Managementreformen in internationalen Organisationen zu erläutern. Führende Wissenschaftler auf diesem Gebiet analysieren die Reformprozesse u. a. in der EU-Kommission, dem Nordischen Ministerrat, der Weltbank, der OECD, der EZB, dem Europäischen Parlament und der UN.

Bitte bestellen Sie bei Ihrer Buchhandlung oder bei Nomos

C07221/2104-37|国-43|www.nomos.de | sabine.horn@nomos.de 